\title{
Teor de Sódio em Refrigerantes com e sem Adição de Açúcar
}

Camila S. Lui, Marina F. B. Souza, Nástia R. A. Coelho, Carla C. B. Machado \& Evilázaro M. O. Castro

O objetivo deste trabalho foi analisar o teor de sódio de refrigerantes com e sem adição de açúcar dos sabores cola, guaraná e limão de duas marcas distintas. Utilizouse a metodologia de fotometria de chama. Puderam-se observar variações intra e entre marcas; os valores mais altos de sódio foram em refrigerantes diet ou zero.

Palavras-chave: fotometria de chama; sódio; refrigerantes.

The objective was to analyze the sodium content of soft drinks with and without added sugar flavors of cola, guarana and lemon in two distinct brands. We used the method of flame photometry. It was observed variations within and between brands; the values were higher in sodium diet sodas or zero.

Key words: flame photometry; sodium; soft Drinks. 


\section{Introdução}

Apesar da atual visibilidade no cenário mundial, no que tange ao desenvolvimento cultural, econômico e científico, o Brasil, em termos de saúde populacional, enfrentou e ainda vem enfrentando o processo mais diferenciado e desafiador no referencial de políticas e programas voltados para a avaliação epistemológica, a qual vem demonstrando grandes mudanças nos padrões de morbimortalidade ${ }^{1,2}$.

O processo de transição nutricional está diretamente relacionado com uma complexa rede de mudanças nos padrões demográfico, socioeconômico e de saúde, envolvendo fatores como urbanização, desenvolvimento da economia, crescimento tecnológico e alterações culturais, sendo estas transformações ocorridas nos três últimos séculos ${ }^{1,2}$.

Ao se analisar dados estatísticos que retratam as referidas variações do processo saúde/doença, tornase possível observar divergências significativas, no que se refere à atual evolução da vida humana, quando comparada com a primeira metade do século XX e, consequentemente do século XIX. As mudanças ocorridas ao longo dos anos refletem a interferência de fatores externos, provenientes de uma progressiva e irreversível globalização, propiciando maior desenvolvimento e, assim, delineando os processos históricos e culturais da população brasileira ${ }^{3,1}$.

Caracterizado, essencialmente, por alterações na estrutura da dieta e na composição corporal dos indivíduos, as modificações no perfil de saúde da população estão associadas à crescente participação da mulher no campo de trabalho; ao fortalecimento da economia; ao aumento nos rendimentos e ao poder de compra da população, acarretando, assim, mudanças no estilo de vida $4,5,6,7,8$.

Partindo deste contexto, é possível correlacionar o crescimento econômico, o fortalecimento da moeda de compra e consequentemente, a viabilização de ampliação do mercado consumidor; contribuindo para as mudanças de hábito, favorecendo o aumento do consumo de alimentos e bebidas industrializadas, propiciando o consumo inadequado de alimentos ricos em sódio, gorduras, carboidratos refinados e com elevado valor energético, podendo comprometer a saúde desde os primeiros anos de vida, quanto na fase adulta; além de estimular a redução do consumo de alimentos in natura ${ }^{9,1}$.

Dentre os produtos industrializados mais consumidos por diferentes grupos e faixas etárias, destaca-se o refrigerante e, de acordo com várias pesquisas, incluindo a Pesquisa de Orçamentos Familiares (POF, 2008-2009), a prevalência de consumo em comparação com a década de 1970, aumentou cerca de $400 \%{ }^{10}$.

Vale ressaltar que refrigerantes são bebidas carbonatadas, não alcoólicas e adicionadas de aromas. Sua formulação se dá a partir de uma mistura de água, gás carbônico, concentrados aromatizados, acrescido de açúcar ou adoçante, além de outras substâncias; podendo ser encontrado em uma grande variedade de sabores. Mesmo sendo produtos de baixo valor agregado, e desconsiderados como itens de primeira necessidade, seu consumo anual per capita no Brasil chega a 70 litros, ficando atrás apenas dos Estados Unidos, com 201 litros, México 147,3 e Alemanha com 82,3 litros. Quanto à preferência de sabores no país, os mais vendidos são cola, com $50,9 \%$, guaraná $24,5 \%$ e o restante dos sabores juntos totalizam $24,6 \%$, já os refrigerantes diet e light atingem cerca de $8,5 \%$ do total consumido $11,12,13$.

Segundo a Associação Brasileira das Indústrias de Refrigerantes e Bebidas não Alcoólicas (ABIR) ${ }^{14}$, a empresa Canadean Liquid Intelligence realizou uma projeção de todas as bebidas não alcoólicas consumidas no Brasil no período de 2004 a 2012, estimadas em milhões de litros por ano. Baseado nos dados fornecidos, em 2004, o Brasil consumiu cerca de 12.713,89 milhões de litros de refrigerante, o que representa $28,2 \%$ do total de bebidas não alcoólicas. Em 2010, o valor passa para 15.368,83 milhões de litros, um total de 27,2\%, 2011 atingirá 15.645,47, estimando um percentual de $26,9 \%$ do consumo de bebidas não alcoólicas e, por fim, 2012 chegando à marca de 15.974 .02 milhões de litros, perfazendo 26,6\%.

Preocupados com esse expressivo aumento de 
consumo, verificou-se a necessidade de avaliar o teor de sódio encontrado em refrigerantes, levandose em conta, que este mineral é um ingrediente fundamental na formulação de alimentos e bebidas industrializadas, com a função de realçar o sabor e conferir maior tempo de conservação. Desta forma, o aumento do consumo de alimentos processados, e a alta concentração de sódio contida nestes produtos, estão contribuindo para a elevação dos níveis pressóricos em crianças e adolescente, bem como em adultos e idosos ${ }^{15,16,17}$.

A importância de se avaliar o consumo e a concentração de sódio em alimentos e bebidas industrializadas está diretamente ligada ao crescimento nos índices de Doenças Crônicas Não Transmissíveis (DCNTs), revelando-se um dos principais desafios de saúde, no que diz respeito ao desenvolvimento global nas próximas décadas. Considerada uma ameaça à qualidade de vida de milhares de pessoas, essas enfermidades também representam aumento nos custos para os sistemas de saúde de todo o mundo, promovendo grande impacto econômico para os portadores, suas famílias e consequentemente a sociedade em geral, atingindo desde países ricos, e principalmente os em desenvolvimento ${ }^{18,19}$.

De acordo com a Organização Mundial de Saúde (OMS), no ano de 2001, imputaram-se as DCNTs um total de 33,1 milhões de óbitos, o que representa 58,5\% da mortalidade mundial, e $45,9 \%$ do quantitativo global de doenças. No Brasil, em 2008, estas enfermidades atingiram o percentual de $62,8 \%$ do total de mortes por causa conhecida, indicando um aumento três vezes maior entre as décadas de 30 e 90; se permaneceramse os avanços, em 2020 as DCNTs serão responsáveis por $73 \%$ dos óbitos e $60 \%$ das doenças globais, tornando-se, assim, a principal causa de incapacidade no mundo ${ }^{20}$.

Compreendida como uma epidemia na atualidade, as DCNTs são definidas como afecções de longa permanência, oscilando entre quadros agudos (piora repentina), e sensível melhora; seus fatores de risco são classificados em não modificáveis, destacando-se idade, sexo, raça, e a hereditariedade, e os modificáveis, incluindo estresse, tabagismo, etilismo, obesidade, dislipidemias, diabetes mellitus e a hipertensão arterial ${ }^{19}$.

Dentre os fatores de risco modificáveis, a Hipertensão Arterial Sistêmica (HAS), vem sendo associada ao expressivo consumo de sódio, baseado em algumas pesquisas que avaliaram o consumo alimentar inadequado, rico em alimentos processados e bebidas industrializadas, bem como, a sua relação com os níveis pressóricos desta população ${ }^{18,19,20}$.

Diante deste cenário, tendo em vista a importância e a contribuição destas avaliações para a saúde da população, faz-se necessária e iminente a continuidade deste tipo de pesquisa, a fim de se analisar os reais valores de concentração de sódio em refrigerantes com e sem adição de açúcar, e estabelecer o paralelo entre a prevalência de consumo e suas implicações para a saúde.

\section{Materiais e Métodos}

\section{AMOSTRAS}

As amostras foram adquiridas no comércio varejista local, sendo 3 amostras de 3 lotes diferenciados para cada um dos sabores, bem como para cada tipo de bebida, (com e sem adição de açúcar). Sendo estabelecido o mesmo critério para ambas as marcas avaliadas, perfazendo-se assim, um total de 36 amostras.

\section{CRITÉRIOS DE INCLUSÃO E EXCLUSÃO}

Foram incluídas no estudo, marcas de refrigerantes que apresentassem os dois tipos de bebidas (com e sem adição de açúcar), para os 4 principais sabores (cola, guaraná, limão e laranja). Das marcas existentes, apenas 2 atenderam aos critérios estabelecidos, porém, para uma delas, não foi possível adquirir o sabor laranja sem adição de açúcar, por não estar disponível no comércio local. Sendo assim, o sabor mencionado foi excluído da presente pesquisa, permanecendo, então, apenas os sabores (cola, guaraná e limão).

\section{DETERMINAÇÃO DE SÓDIO NA+}

Conhecido como método original, a fotometria de chama é utilizada principalmente para análise de metais alcalinos como sódio, sendo esta a mais simples, rápida e de alta sensibilidade das técnicas analíticas baseadas em espectroscopia atômica. 
Com base no exposto acima, as amostras foram avaliadas por fotometria de chama, com aparelho da marca QUIMIS - modelo (Q398M), disponibilizado pela Pontifícia Universidade Católica de Goiás, adaptando-se à metodologia descrita pelo Instituto Adolfo Lutz, para determinação de sódio em águas, haja vista que não foi encontrada metodologia específica para análise do teor de sódio em refrigerantes; ajustando-se à sensibilidade do equipamento e seguindo seus padrões de adequação.

\section{PREPARAÇÃO DO PADRÃO}

Pesou-se em um béquer de $50 \mathrm{~mL}$ a massa de $0,6355 \mathrm{~g}$ de cloreto de sódio seco em estufa à $140^{\circ} \mathrm{C}$ por 3 horas, e resfriouse em dessecador na temperatura ambiente; finalizado o resfriamento, transferiu-se para um balão volumétrico de 250 $\mathrm{mL}$, completou o volume com água deionizada, e obteve-se, assim, uma solução com concentração de $1 \mathrm{mg} / \mathrm{mL}$ ou 1000 $\mathrm{mg} / \mathrm{L}$.

\section{PREPARAÇÃO DA CURVA DE CALIBRAÇÃO}

A partir da solução padrão de $1000 \mathrm{mg} / \mathrm{L}$, preparou-se uma solução de $100 \mathrm{mg} / \mathrm{L}-1$, transferindo $10 \mathrm{~mL}$ para um balão volumétrico de $100 \mathrm{~mL}$, previamente calibrado, e completou o volume com água deionizada.

Após essa etapa, pipetou-se $5 \mathrm{~mL}$ da solução padrão de $100 \mathrm{mg} / \mathrm{L}-1$ em um balão volumétrico de $50 \mathrm{~mL}$, alcançandose uma solução com concentração de $10 \mathrm{mg} / \mathrm{L}-1$ de sódio.

Fez-se a calibração do fotômetro de chama, utilizandose água deionizada como branco, e realizou-se a leitura das soluções padrão. Após a leitura de cada amostra, verificouse o zero da escala do aparelho com água deionizada, construindo um gráfico de calibração com intensidade de emissão em função da concentração de íons sódio em mg/L.

\section{PREPARAÇÃO DAAMOSTRA}

Adicionou-se no erlenmeyer de $125 \mathrm{~mL}, 50 \mathrm{~mL}$ da amostra e, em seguida, levou-se ao banho ultrassônico por 30 minutos para a remoção dos gases. Transferiu-se quantitativamente para 3 balões volumétricos de $50 \mathrm{~mL}$ previamente calibrados, $2,5 \mathrm{~mL}$ da amostra degaseificada e completou o volume até o traço para aferição, com água deionizada, homegeneizando-as uma a uma.

As análises foram realizadas em triplicata da mesma amostra, repetindo o ensaio em 3 lotes diferenciados, objetivando melhor padrão e precisão do estudo.

\section{LEITURA DAAMOSTRA}

As amostras foram lidas por aspiração do conteúdo de cada balão, determinando a concentração de íons sódio em $\mathrm{mg} / \mathrm{L}$, utilizando-se água deionizada para limpeza e eliminação de interferências entre cada leitura realizada.

O banco de dados foi construído no Software Excel do Pacote Office for Windows, versão 2007, e análise estatística foi realizada no Software Instat versão 2.01, utilizando-se o Teste T não pareado, considerando o intervalo de confiança de 95\% nas diferenças das médias, atribuindo-se diferenciação de dados, quando os valores de (p) forem julgados baixo; sendo esta uma análise descritiva, de variáveis qualitativas e quantitativas, representadas por meio de gráficos.

\section{Resultados e Discussão}

A determinação de sódio foi realizada em duas marcas distintas A e B, analisando-se os sabores cola, guaraná e limão, contemplando os tipos de bebida normal e zero. De acordo com os dados obtidos, os refrigerantes apresentaram uma variação de $35 \mathrm{mg} /$ sódio/L a $186 \mathrm{mg} /$ sódio/L, perfazendo uma média de $86 \mathrm{mg} /$ sódio/L. A bebida normal demonstrou valores entre $35 \mathrm{mg} /$ sódio/L e $78 \mathrm{mg} /$ sódio/L, indicando uma média de $60 \mathrm{mg} / \mathrm{sódio} / \mathrm{L}$, enquanto o zero entre $76 \mathrm{mg} /$ sódio/L e $186 \mathrm{mg} /$ sódio/L, com uma média de $112 \mathrm{mg} /$ sódio/L (Figura 1).

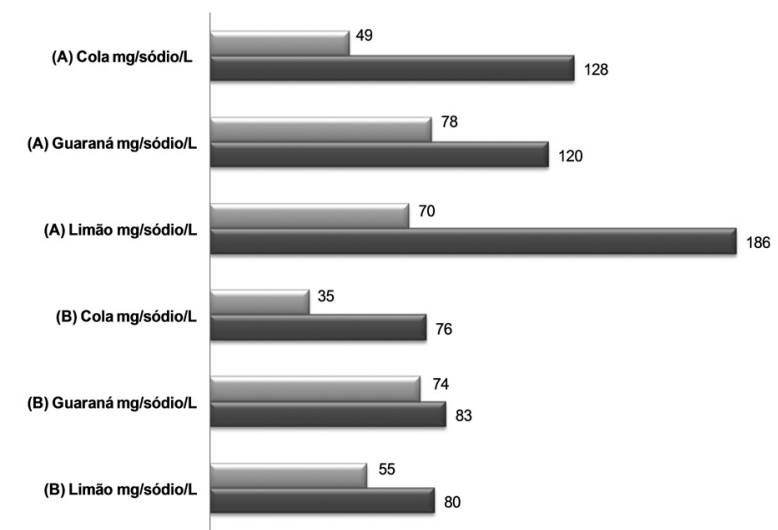

Figura 1. Análise de teor de sódio de refrigerantes normal e zero marcas A e B 
No que se refere ao comparativo do teor de sódio em refrigerantes com adição de açúcar (Figura 2), observou-se que dentre as marcas avaliadas, a marca A apresentou maior concentração de sódio para todos os sabores em relação à marca $\mathrm{B}$, (cola marca $\mathrm{A}-\mathrm{mg} / \mathrm{L}$ ) média $=49,11 \pm 1,453$, (cola marca $B-\mathrm{mg} / \mathrm{L}$ ) média $=35,6666 \pm 1,323$, apontando uma diferença entre médias de $-13,444 \mathrm{mg} / \mathrm{L}(\mathrm{p}<0,0001)$, evidenciando divergências extremamente significantes, assim como, (limão marca $\mathrm{A}-\mathrm{mg} / \mathrm{L}$ ) média $=70,444 \pm 1,667$, (limão marca $\mathrm{B}-\mathrm{mg} / \mathrm{L}$ ) média $=55,777 \pm 1,563$, diferença de $-14,667 \mathrm{mg} / \mathrm{L}(\mathrm{p}<0,0001)$ e, por fim, o sabor (guaraná marca $\mathrm{A}-\mathrm{mg} / \mathrm{L}$ ) média $=78,666 \pm 2$ (guaraná marca $\mathrm{B}$ ) média $=75,555 \pm 2,068$, diferença de $-3,111 \mathrm{mg} / \mathrm{L}(\mathrm{p}=0,0051)$, mesmo sendo menor que os anteriores, também apresenta distinção de valores muito significativos. Por outro lado, o paralelo entre os desvios padrão, não denotou acepção entre as duas marcas, demonstrando, assim, uma semelhança quanto ao grau de padronização.

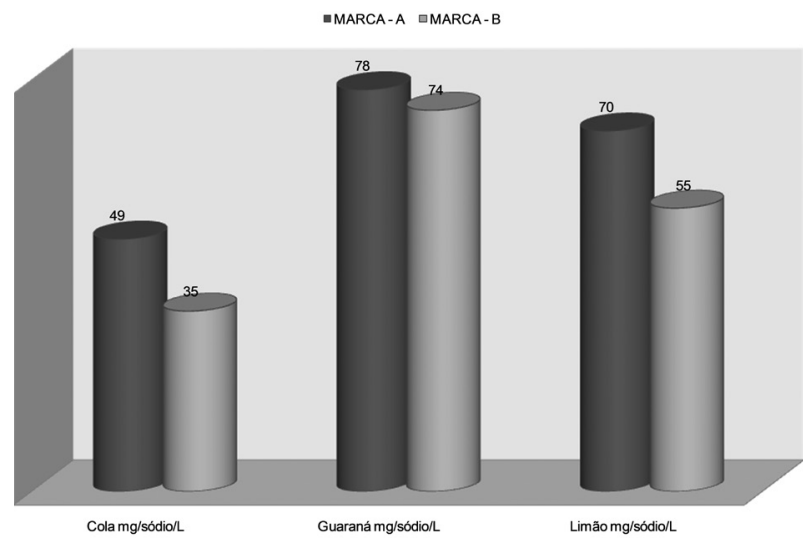

Figura 2. Comparativo do teor de sódio em refrigerantes com adição de açúcar - marcas A e B

Em se tratando da concentração de sódio em refrigerantes sem adição de açúcar, a prevalência também foi maior na marca A, manifestando uma discrepância para todos os sabores; (cola marca $\mathrm{A}-\mathrm{mg} / \mathrm{L}$ ) média = $128,222 \pm 2,108$, (cola marca B $-\mathrm{mg} / \mathrm{L}$ ) média $=76,666$ $\pm 0,500$, com diferença de $-51,556(\mathrm{p}<0,0001)$, (guaraná marca $\mathrm{A}-\mathrm{mg} / \mathrm{L}$ ) média $=120 \pm 2,646$, (guaraná marca $\mathrm{B}-\mathrm{mg} / \mathrm{L})$ média $=82,888 \pm 2,315$, diferença de $-37,111$ ( $\mathrm{p}<0,0001$ ), e o sabor (limão marca $\mathrm{A}-\mathrm{mg} / \mathrm{L}$ ) média $=186 \pm 1,732$, (limão marca $\mathrm{B}-\mathrm{mg} / \mathrm{L})$ média $=80 \pm$ 1,581, divergência entre médias de $-106(\mathrm{p}<0,0001)$ todos considerados extremamente significativos quanto à diferença no quantitativo de sódio. Já os valores de (p) no comparativo dos desvios padrão, apontam uma padronização em ambas as marcas para os sabores guaraná e limão, enquanto que o sabor cola apresenta um ( $\mathrm{p}=0,0002)$ valor este de extrema significância, sugerindo, assim, a falta de padronização para uma das marcas. (Figura 3).

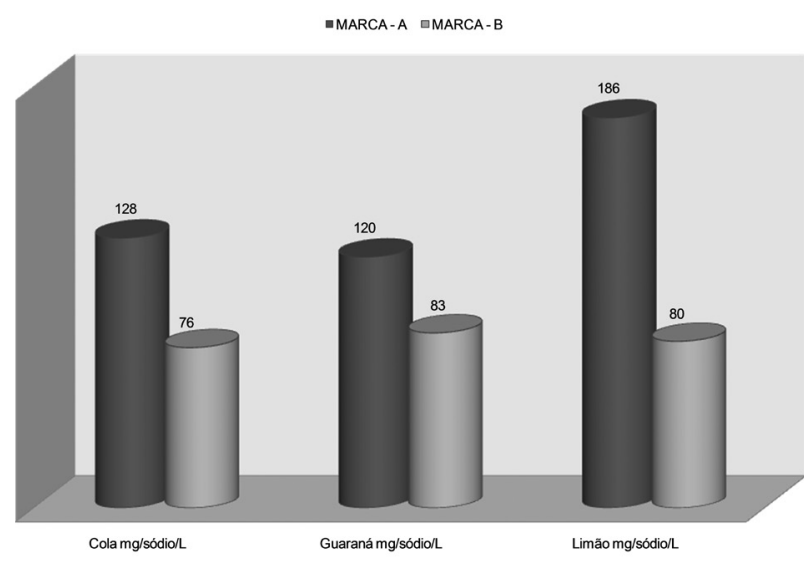

Figura 3. Comparativo do teor de sódio em refrigerantes sem adição de açúcar - marcas A e B

De acordo com a (Figura 4), a comparação feita entre valores de sódio, contidos no rótulo dos refrigerantes da marca A, para ambas as bebidas (normal e zero) e os encontrados neste estudo, apresentaram discordâncias, entretanto, os valores analisados foram menores do que o informado no rótulo do fabricante.

Já a informação nutricional de sódio dos refrigerantes da marca $B$, além de evidenciarem divergências de resultados para quase todos os sabores e os tipos de bebida, apontaram diferenças significativas entre os rótulos e as análises realizadas (Figura 5). 


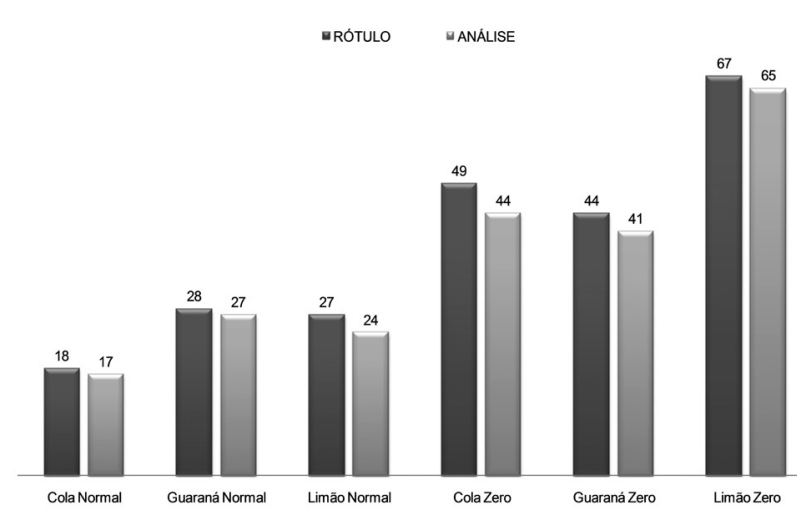

Figura 4. Comparativo do teor de sódio marca $\mathrm{A}$ - rótulo e análise valores de sódio $\mathrm{mg} / \mathrm{Lata}(350 \mathrm{~mL})$

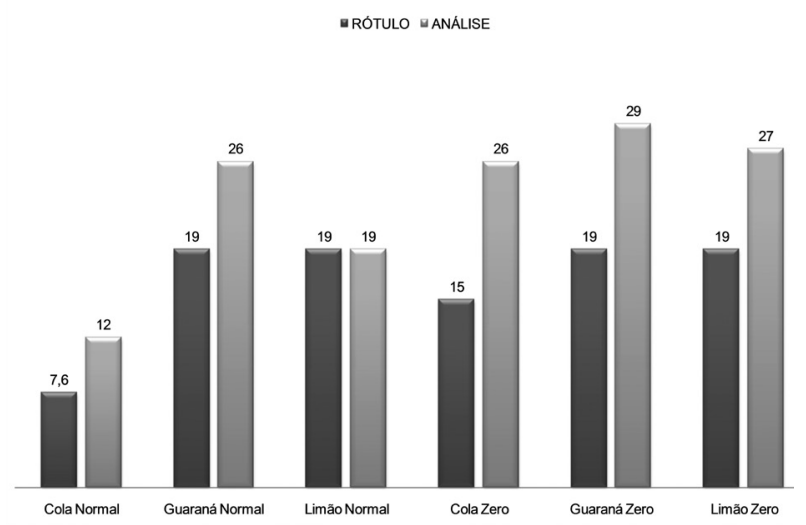

Figura 5. Comparativo do teor de sódio marca B - rótulo e análise valores de sódio $\mathrm{mg} / \mathrm{Lata}(350 \mathrm{~mL})$

As concentrações de sódio encontradas neste estudo evidenciaram uma variação geral entre todos os sabores e bebidas de $35 \mathrm{mg} / \mathrm{sódio} / \mathrm{L}$ a $186 \mathrm{mg} / \mathrm{sódio} / \mathrm{L}$, com média de $86 \mathrm{mg} / \mathrm{sódio} / \mathrm{L}$; indicativos estes que corroboram com pesquisas realizadas ${ }^{20,23}$, as quais apresentaram variantes e médias aproximadas, sendo $20 \mathrm{mg} /$ sódio/L a $151 \mathrm{mg} / \mathrm{sódio} / \mathrm{L}$, com média de $97 \mathrm{mg} /$ sódio/L, e 28mg/ sódio/L a $156 \mathrm{mg} / \mathrm{sódio/L} \mathrm{com} \mathrm{média} \mathrm{de} 95 \mathrm{mg} /$ sódio/L sucessivamente.
Os estudos citados destacam a divergência de teor de sódio encontrado nas bebidas com e sem adição de açúcar, os quais revelam que as bebidas denominadas zero ou diet chegam apresentar cerca de duas vezes a concentração de sódio contida nos refrigerantes ditos "normais", ou seja, com adição de açúcar. O que também pôde ser comprovado na presente pesquisa, a qual identificou diferenças significativas entre os tipos de bebidas, destacando percentuais como o sabor cola da marca B com um acréscimo de $117 \%$ em relação ao refrigerante com açúcar, o cola da marca A com 161\% e o sabor limão também da marca A com $166 \%$ a mais do teor de sódio encontrado na versão normal.

Tais resultados podem ser explicados pelos aditivos que compõem a formulação deste tipo de bebida, os quais apresentam finalidades específicas de conservação como o benzoato de sódio ou ácido benzoico que visa inibir o desenvolvimento de micro-organismos, assim como os edulcorantes, ciclamato de sódio e sacarina de sódio, capazes de conferir sabor doce, substituindo a sacarose; além de estabilizantes como o citrato de sódio, utilizado com a função de assegurar características físicas, emulsão e suspensão dos produtos ${ }^{21,22}$.

Ao se avaliarem os índices encontrados por Ferrari e Soares $(2003)^{23}$, estabeleceu-se uma relação entre o consumo de refrigerante e a quantidade de sódio ingerida; na qual o consumo médio mensurado em $259 \mathrm{~mL}$ por dia, representa a ingestão de $19 \mathrm{mg}$ de sódio diário oriundos do refrigerante formulado com açúcar. Em contrapartida, os consumidores que optarem pela bebida à base de adoçante, estarão ingerindo diariamente $39 \mathrm{mg}$ de sódio. Se correlacionados estes valores com o alto índice de consumo de refrigerantes no Brasil, estimando-se o quantitativo de (2L/dia), os resultados saltam para $147 \mathrm{mg}$ de sódio para os refrigerantes com adição de açúcar, e $300 \mathrm{mg}$ para as bebidas diet ou zero.

Com base nos valores encontrados no presente estudo, o consumo de refrigerantes com adição de açúcar, levando-se em conta a menor determinação de sódio avaliada, representa a ingestão de $35 \mathrm{mg} /$ sódio/L, enquanto os adeptos do refrigerante diet consumirão o quantitativo de $186 \mathrm{mg} /$ sódio/L. Conforme citado, se for estabelecido o mesmo paralelo entre percentuais de consumo, considerando-se o montante de 2 litros diários, os indicativos de teor de sódio alcançarão $70 \mathrm{mg} / 2 \mathrm{~L}$, 
representando as bebidas com adição de açúcar e 370 $\mathrm{mg} / 2 \mathrm{~L}$ para os refrigerantes diet ou zero.

Essa estimativa de consumo não pode ser considera utópica se for comparada com pesquisas realizadas acerca do consumo e das preferências alimentares de grupos distintos, como mostra o estudo feito com 334 adolescentes de uma escola particular da cidade de Teresina/PI, o qual se avaliou o consumo semanal de três grupos alimentares, os construtores, energéticos e reguladores; do grupo dos energéticos, predominou o consumo de refrigerantes, chegando atingir 94,70\% da preferência dos adolescentes do sexo masculino, e $87,78 \%$ do sexo feminino. Ao ser comparado com outro alimento, a preferência masculina pela bebida energética, superou o consumo de arroz que foi de $91,94 \%$.

Assim, como a pesquisa realizada em 1423 alunos de escolas estaduais e municipais da cidade de Niterói/RJ, a qual identificou um consumo significativo de refrigerante; demonstrando que, dos alunos que só consumiam a bebida normal, atingiu o percentual de $90,8 \%$, dos que consumiam normal e light, 5,5\% e dos light e diet, 3,7\%. Quanto à frequência deste consumo, de 601 participantes do sexo masculino 39,9\% afirmaram consumir de 2-4 vezes por semana, e 15\%, no mínimo, 2 vezes ou mais por dia; do sexo feminino, das 646 componentes da amostra, $42,2 \%$ consomem refrigerante de $2-4$ vezes por semana, e $13 \% 2$ vezes ou mais diariamente. Os índices gerais encontrados superam o consumo de leite integral que chegou à marca de $86,7 \%$ e de iogurte integral $81,3 \% .^{20}$.

Os resultados apresentados acabam por confirmar a crescente evolução no consumo de refrigerantes no país, assim como demonstrado na última pesquisa de Vigilância de Fatores de Risco e Proteção para Doenças Crônicas por Inquérito Telefônico ${ }^{20}$, a qual indica que o consumo de refrigerantes, 5 vezes na semana, representa $27,9 \%$ dos fatores de risco à saúde; ao analisar o consumo segundo o sexo $31,5 \%$ está representado por homens, e $23,3 \%$ por mulheres; de acordo com análise feita entre as capitais brasileiras, incluindo o Distrito Federal, o menor índice foi em Natal com 12\% e o maior em Porto Alegre, atingindo $39 \%$ da população entrevistada ${ }^{19}$.

Inserido neste contexto, é possível estabelecer uma relação entre consumo de refrigerantes e sua possível implicação na alteração dos níveis pressóricos. Conforme observado no estudo realizado com 564 adolescentes de
20 escolas municipais de Caxias do Sul/RS, em que a prevalência de hipertensão encontrada foi de 21,7\%. Em relação ao consumo alimentar destes escolares, dentre os valores mais significativos com frequência de consumo, sendo de (1-2 vezes na semana), o mais consumido dentre todos, foi o refrigerante com $41 \%{ }^{16}$.

Quanto ao comparativo realizado acerca dos quantitativos de sódio, informados nos rótulos dos refrigerantes da marca $\mathrm{A}$, para os dois tipos de bebida (normal e zero), apresentaram-se inferiores aos valores determinados no presente estudo, podendo essa diferença de resultados ser atribuída à possível divergência de métodos de análise.

Já a discrepância encontrada entre a informação nutricional da marca $\mathrm{B}$ e as concentrações de sódio analisadas nessa pesquisa, evidenciaram valores bem acima do permitido pela legislação vigente ${ }^{22}$, a qual estabelece a tolerância de $20 \%$ a mais dos quantitativos de nutrientes informados no rótulo; produtos com os valores encontrados fora desse parâmetro poderão ser condenados. Sendo assim, faz-se necessária uma revisão das análises do teor de sódio por parte da indústria, bem como a reformulação da rotulagem desses produtos.

Em contrapartida, as altas concentrações de sódio utilizadas pela indústria alimentícia estão amparadas pela $\left(\mathrm{RDC} \mathrm{n}{ }^{\mathrm{o}} 24\right)^{24}$, que atribui elevada quantidade de sódio, somente para os alimentos que possuírem valores iguais ou superiores a $400 \mathrm{mg}$ /sódio por 100 gramas ou 100 mililitros, sendo esse na forma como estiver exposto à venda. Resguardados os devidos parâmetros, observa-se o quão ampla e concedente se mostra a resolução vigente.

\section{Conclusão}

Os resultados demonstraram diferenças no teor de sódio para todos os sabores, evidenciando um acréscimo relevante para os refrigerantes diet ou zero, destacando-se principalmente os sabores cola e limão. Das duas marcas avaliadas, a marca $\mathrm{A}$ apresentou maior concentração de sódio para ambas as bebidas em todos os sabores em relação à marca $\mathrm{B}$. O comparativo estabelecido entre rotulagem e as análises realizadas, refletiram divergência de valores, com discrepâncias significativas em quase todos os refrigerantes da marca B. Com base nos resultados, faz-se necessário o aprofundamento desse tipo de estudo, visando estabelecer melhor a 
relação entre a concentração de sódio ingerida frente à frequência de consumo destas bebidas industrializadas, e o quanto esses valores representam no total de sódio ingerido diariamente. Ainda partindo desse princípio, é imprescindível a iminência de mudanças na legislação, a fim de se estabelecer redução do teor de sódio em alimentos e bebidas industrializadas, bem como o rigor no cumprimento dessas leis.

\section{Referências}

1. Batista Filho, M., Batista, L.V. Transição alimentar/nutricional ou mutação antropológica. Ciência e Cultura, 2010; 62(4): 26-30. doi: 0009-6725.2. SOUZA, E. B. Transição nutricional no Brasil: análise dos principais fatores. Cadernos UniFOA. 2010; (13): 49-53.

2. Batista Filho, M., Rissin, A. A transição nutricional no Brasil: tendências regionais e temporais. Cadernos de.Saúde Pública. 2003; 19(1): 181-191.

3. Anjos, M.A. Retrospectiva da economia brasileira nos últimos 45 anos: industrialização, desenvolvimento, crises, políticas neoliberais e injustiça social caracterizam a evolução da economia do país. Revista FAE Business. 2002; (4): 18-21.

4. Probst, E.R. A evolução da mulher no mercado de trabalho. Revista do Instituto Catarinense do Pós-Graduação [internet]. 2003, v. 2, jan-jun, p. 1-8, 2003.

5. Hoffman, R., Leone, E. T. Participação da mulher no mercado de trabalho e desigualdade da renda domiciliar per capita no Brasil: 1981-2002. Revista Nova Economia. 2004; 14(2): 35-58.

6. Tardido A.P., Falcão M. C. O impacto da modernização na transição nutricional e obesidade. Revista Brasileira de Nutrição Clínica. 2006; 21(2): 117-124.

7. Instituto Brasileiro De Geografia E Estatística - IBGE (Brasil). Indicadores IBGE - pesquisa mensal de emprego: principais destaques da evolução do mercado de trabalho nas regiões metropolitanas abrangidas pela pesquisa: Recife, Salvador, Belo Horizonte, Rio de Janeiro, Porto Alegre (2003-2010). Rio de Janeiro, RJ, 2010. 215p.

8. Carvalho, C. M. R. G., Nogueira, A. M. T., Teles J. B. M., Paz, S. M. R., Souza, R.M. L. Consumo alimentar de adolescentes matriculados em um colégio particular de Teresina, Piauí, Brasil. Revista Nutrição. 2001; 14(2): 85-93.

9. Instituto Brasileiro De Geografia E Estatística - Ibge (Brasil). Pesquisa de orçamentos familiares (2008-2009): despesas, rendimentos e condições de vida. Rio de Janeiro, RJ, 2010. 222p

10. Tochini, R. P., Nisida, A. L. A. C. Industrialização de Refrigerantes - manual. $1^{\text {a }}$ ed. Campinas. Instituto de Tecnologia de Alimentos - ITAL; 1995.

11. Acnielsen. Alimentos \& Bebidas. Relatório Executivo de
Notícias, 2004. Disponível em: $<$ http://www.acnielsen.com. br/reports/documents/OsProdutosMaisQuentesdoMudoAlimentoseBebidas2004.pdf $>$. Acesso em: 10 out. 2011, $23: 18: 32$.

12. Rosa, S. E. S., Consenza, J. P., Leão, L. T. S. O panorama do setor de bebidas no Brasil. BNDES Setorial, Rio de Janeiro, n. 23, p. 101-150, 2006.

13. Associação Brasileira Das Indústrias De Refrigerantes $E$ De Bebidas Não Alcoólicas - Abir. Projeção de bebidas não alcoólicas 2004 a 2012, 2012.

14. Hoffman M., Silva, A. C. P., Siviero, J. Prevalência de hipertensão arterial sistêmica e inter-relações com sobrepeso, obesidade, consumo alimentar e atividade física, em estudantes de escolas municipais de Caxias do Sul. Pediatria (São Paulo). 2010; 32 (3): 163-172.

15. Brasil. Ministério da Saúde. Agência Nacional de Vigilância Sanitária - Anvisa. Perfil nutricional dos alimentos processados. (Informe Técnico n.42/2010). Brasília, DF, 2010. 52p.

16. Brasil. Ministério da Saúde. Diretrizes e recomendações para o cuidado integral de doenças crônicas não transmissíveis: promoção da saúde, vigilância, prevenção e assistência. (Série B. Textos Básicos de Saúde - Série Pactos pela Saúde 2006, v. 8). Brasília, DF, 2008. 72p.

17. Brasil. Ministério da Saúde. Coordenação nacional de hipertensão e diabetes - CNHD. Departamento de atenção básica saúde da família. Brasília, DF, 2011. 54p.

18. Mendham J., Denney, R.C., Barnes, J. C., Thomas, M. J. K. In: Vogel. Espectroscopia de emissão atômica. $6^{\text {a }}$ ed. Rio de Janeiro: LTC S.A; 2002.

19. Brasil. Ministério da Saúde. Vigilância de fatores de risco e proteção para doenças Crônicas por inquérito telefônico (VIGITEL/2009). Brasília, DF, 2010. 152p. Disponível em < http://bvsms.saude.gov.br/bvs/publicacoes/vigitel_2010.pdf $>$. Acesso em 22 mar. 2013.

20. Palha, P.G. Tecnologia de refrigerantes. Rio de Janeiro: AmBev, 2005.

21. Brasil. Ministério da Saúde. Agência Nacional de Vigilância Sanitária (Brasil). Resolução nº 360, de 23 de Dezembro de 2003. Brasília, DF, 2003.

22. Ferrari, C. C., Soares, L. M. V. Concentrações de sódio em bebidas carbonatadas nacionais. Revista Ciência e Tecnologia de Alimentos. Campinas. 23(3). 414-417. set.-dez. 2003.

23. Brasil. Ministério da Saúde. Agência Nacional de Vigilância Sanitária. Resolução n ${ }^{\circ}$ 24, de 15 de Junho de 2010. Dispõe sobre a oferta, propaganda, publicidade, informação e outras práticas correlatas cujo objetivo seja a divulgação e a promoção comercial de alimentos considerados com quantidades elevadas de açúcar, de gordura saturada, de gordura trans, de sódio, e de bebidas com baixo teor nutricional, nos termos desta Resolução, e dá outras providências. Brasília, DF. 2013. 


\section{Camila S. Lui*, Marina F. B. de Souza, Nástia R. Almeida Coelho, Carla C. B. Machado \& Evilázaro M. O. Castro}

Pontifícia Universidade Católica de Goiás, Departamento de Matemática, Física, Química e Engenharia de Alimentos. CP. 86, Setor Universitário. Goiânia, Goiás. CEP 74605-010

* stramandinoli_lui@hotmail.com 
